\title{
Generating complex three-dimensional stimuli (Greebles) for haptic expertise training
}

\author{
THOMAS W. JAMES \\ Vanderbilt University, Nashville, Tennessee \\ and Indiana University, Bloomington, Indiana \\ DANIEL W. SHIMA \\ Vanderbilt University, Nashville, Tennessee \\ MICHAEL J. TARR \\ Brown University, Providence, Rhode Island \\ and \\ ISABEL GAUTHIER \\ Vanderbilt University, Nashville, Tennessee
}

\begin{abstract}
An apparatus is described that accurately measures response times and video records hand movements during haptic object recognition using complex three-dimensional (3-D) forms. The apparatus was used for training participants to become expert at perceptual judgments of 3-D objects (Greebles) using only their sense of touch. Inspiration came from previous visual experiments, and therefore training and testing protocols that were similar to the earlier visual procedures were used. Two sets of Greebles were created. One set (clay Greebles) was hand crafted from clay, and the other (plastic Greebles) was machine created using rapid prototyping technology. Differences between these object creation techniques and their impact on perceptual expertise training are discussed. The full set of these stimuli may be downloaded from www.psychonomic.org/archive/.
\end{abstract}

We typically recognize objects in our environment by their shape using vision, but we can also recognize an object's shape when our eyes are closed, using our other senses. Perhaps the most apparent example of such a process is recognition using our sense of touch. Before the graphics capabilities of computers were developed to today's level and became readily available, visual object recognition experiments, like tactile experiments, required the use of elaborate apparatuses to maintain a degree of control over the stimulus and the response. Now, visual object recognition experiments are almost entirely conducted using images displayed on computer screens, eliminating the need for any apparatus besides the computer. There have been somewhat analogous technological advances made in the domain of tactile perception, but unlike the common computer monitor, this technol-

This research was supported by grants from the NSF (to I.G.), JSMF (to I.G.), and CIHR (to T.W.J.). Anjali Shah and Mark Kendall provided assistance with data collection. Karin Harman James and other members of the Perceptual Expertise Network (PEN) provided valuable discussions, insights, and comments on this project and manuscript. Jerome Harris facilitated the production of the rapid prototypes. Correspondence relating to this article may be sent to T. W. James, $1101 \mathrm{E}$. Tenth St., Bloomington, IN 47405 (e-mail: thwjames@indiana.edu).

Note-This article was accepted by the previous editor, Jonathan Vaughan. ogy is not widely available. For instance, a Phantom (SensAble Technologies, www.sensable.com) is an automated device that can deliver a specified tactile perceptual stimulus to a single digit (for examples of its use, see Ernst \& Banks, 2002; Ernst, Banks, \& Bülthoff, 2000). Although Phantoms are a marvelous achievement, their capabilities are not yet adequate for addressing questions involving complex tactile shape recognition. Yet, despite the inherent difficulty involved with studying tactile object recognition, many experiments have been designed to investigate how shape and other attributes of objects are processed by the somatosensory system (for a review, see Heller, 2002; for examples, see Kilgour \& Lederman, 2002; Klatzky \& Lederman, 1995) and where in the brain these processes occur (Amedi, Jacobson, Hendler, Malach, \& Zohary, 2002; Hadjikhani \& Roland, 1998; James et al., 2002; Zangaladze, Epstein, Grafton, \& Sathian, 1999).

There are some unique impediments to overcome when designing a tactile perception experiment. For instance, because the stimuli are actual physical objects (unless a device such as the Phantom described above is used), the timing of both the stimulus onset and the stimulus duration (and, consequently, the response time) are difficult to control. Some apparatuses have been developed that do allow accurate control of these parameters (Klatzky \& Lederman, 1995), but they are typically used to deliver two-dimensional (2-D) or simple 3-D stimuli, not com- 
plex 3-D objects. In addition, because of the sequential nature of tactile exploration, hand movements made during exploration are often recorded on videotape for later analysis. This type of analysis is extraordinarily time consuming, but can give valuable insights (for example, see Lederman \& Klatzky, 1993). Measuring movement parameters and recording hand manipulations could be achieved using a 3-D position recording device, such as an Optotrak. This technique is frequently used for the accurate analysis of ballistic grasping movements. Applicability of these recording techniques to complex exploratory movements, however, has yet to be determined and will largely depend on whether useful analysis algorithms can be developed. Finally, there is a tradeoff between the accuracy of the technique for making the tactile stimuli and the complexity of the stimuli that are made. Single-part tactile stimuli that vary along such stimulus dimensions as size and even curvature can usually be machined with great accuracy (Hadjikhani \& Roland, 1998; Prather \& Sathian, 2002; Servos, Lederman, Wilson, \& Gati, 2001). In contrast, multipart stimuli that vary along less explicit dimensions must usually be made by hand (for example, see James et al., 2002), which introduces a certain amount of undesirable variability.

A description follows of an experimental setup that allows considerable control of stimulus and response timing and more efficient video recording of hand movements yet also maintains the ecological integrity of a 3-D object exploration task. In addition, stimuli can be made using a technique, rapid prototyping, that creates complex 3-D plastic forms with high accuracy (for example, see Friedman, Spetch, \& Lank, 2003). A participant under this setup can use one- or two-handed exploration because responses can be made with foot-triggered buttons. Stimulus onset is determined using an infrared detector, which senses imminent contact of the hand with the object. This same infrared detector is also used to engage and disengage a digital video recorder, creating video clips that capture only the discrete time intervals when meaningful exploratory hand movements are being performed, making off-line movement analysis more efficient.

Inspiration for the stimuli and procedures described here came from research on visual perceptual expertise that investigated changes in behavioral performance and brain activation resulting from expertise training with novel objects (Gauthier \& Tarr, 1997; Gauthier, Tarr, Anderson, Skudlarski, \& Gore, 1999; Gauthier et al., 2000; Gauthier, Williams, Tarr, \& Tanaka, 1998; Rossion, Gauthier, Goffaux, Tarr, \& Crommelinck, 2002). These studies were motivated in part by the claim that selective activation of an area in the fusiform gyrus by visually presented faces was in fact specific only to faces. The studies were designed to test the hypothesis that factors other than object geometry, such as the amount of experience with the objects and the type of classification task performed on the objects, influenced activation in the fusiform gyrus. In these studies, participants were trained extensively to identify novel objects, "Greebles," at both group (or family) and individual levels. Greebles are biological-looking novel objects composed of a body and several appendages (Figure 1). Families of Greebles share the same body shape, and individuals within each family differ from each other in the shape of their appendages. With training, participants were able to associate individual and family names with the Greeble stimuli. With extensive training, several behavioral and neural changes occurred. For instance, verifying a Greeble's membership in a particular family was initially quicker
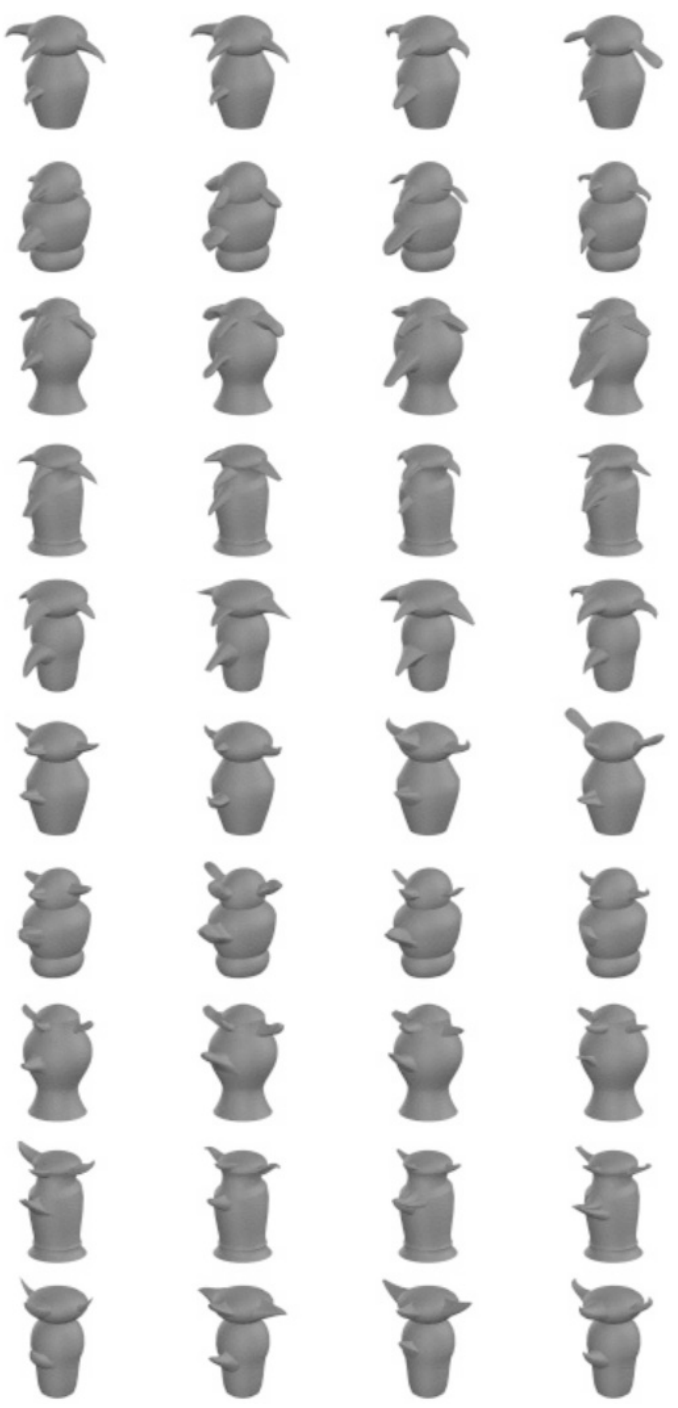

Figure 1. 2-D images of the Greebles-2-D renderings of the 3-D volumetric computer models of the entire set of Greebles. All Greebles share a common configuration of parts. In each row are individual Greebles from the same group based on classification into two independent categories. Greebles from the same family category share the same body shape. Each family contains both Ploks and Glips, which are indicated by the orientation (down or up) of the four appendages. The top five rows are Ploks, and the bottom five rows are Glips. Individuals within a family can differ in the shapes of all four appendages. 
than verifying its individual name, but this difference disappeared during training. Greeble experts could learn to individuate novel exemplars of this class faster than novices could (Gauthier et al., 1998), and they also processed Greebles in a more holistic manner than did novices - that is, experts had more difficulty selectively attending to a single part of these objects (Gauthier \& Tarr, 2002). Training was also related to changes in neural activation; a region of the posterior fusiform gyrus showed increased activation for Greebles relative to control objects associated with the acquisition of Greeble expertise (Gauthier et al., 1999). These changes in fusiform gyrus activity to Greebles were correlated with the increase in holistic processing (Gauthier \& Tarr, 2002). Therefore, an intriguing question is whether these changes are restricted to training in the visual modality?

The studies described above, which employed perceptual expertise training with novel objects, were conducted using visual training paradigms. Although we usually use vision when attempting to recognize objects in the environment, we are also exceptionally skilled at recognizing objects using our sense of touch (Klatzky, Lederman, \& Metzger, 1985). This similarity between the sensory modalities seems to stem partially from the fact that both systems are able to process the 3-D geometrical structure of objects. In fact, there is evidence that the two systems may share a common neural substrate that is responsible for computing object form (Amedi et al., 2002; James et al., 2002). Thus, it is quite possible that tactile and visual perceptual expertise share the same behavioral and neural markers. Recall, though, that one marker of visual perceptual expertise was a shift to a more holistic processing strategy (Gauthier \& Tarr, 2002). Although there are several similarities between the haptic and visual systems, one definite difference is the speed and accuracy with which the visual system can move the high-acuity portion of its receptor surface. A saccadic eye movement can be planned and executed in under $200 \mathrm{msec}$, whereas moving the fingers to a new location of an object takes much longer. Furthermore, because of mechanical constraints, the haptic system cannot always bring lower acuity receptors (such as the palm) to bear on an object that is being explored with the fingertips, whereas peripheral vision receives an obligatory coarse input from the entire scene. Also unlike the visual system, the haptic system is able to manipulate its highacuity receptor surfaces independently, with the two hands being completely independent of each other and the fingers being somewhat independent of the thumbs, providing approximately four independent sources of exploratory information. The visual system's two fovea, on the other hand, are almost always yoked, providing only one source of information.

The speed with which the visual system is able to analyze sequentially an object and/or supplementary information from the periphery may be critical to its holistic processing capabilities; in this case, it seems unlikely that the haptic system would show the same behavioral marker for perceptual expertise. On the other hand, independence of the input from each fingertip may make up for slower movement times in some tasks, and in this case, we may expect the haptic system to show the same behavioral marker for perceptual expertise as the visual system. Therefore, studying haptic perceptual expertise not only informs us about the learning capabilities of the haptic system, but also has the potential to constrain hypotheses about how the haptic and visual systems process similar information.

\section{Tactile Stimuli}

Clay stimuli. The tactile stimuli were 30 novel objects, called "Greebles." In previous visual experiments, 3-D computer models of the Greebles were rendered as 2-D bitmaps (Figure 1), but for tactile presentation, real objects were needed. We created 3-D Greebles using two methods: We first sculpted them from clay (Figure 2), but later also built them using a technology called rapid prototyping (see the next section and Figure 3). Thirty clay Greebles were hand sculpted using air-drying clay by referring to the rendered 2-D images as templates. The resulting set of Greebles had heights that ranged between 7.5 and $10.5 \mathrm{~cm}$, with a mean height of $9.3 \mathrm{~cm}$; widths that ranged between 3.0 and $5.5 \mathrm{~cm}$, with a mean width of $4.6 \mathrm{~cm}$; and depths that ranged between 7.5 and $10.5 \mathrm{~cm}$, with a mean depth of $9.0 \mathrm{~cm}$. Because the clay Greebles were hand prepared, imperfections and differences between the clay models and the computer models were expected. For our particular training paradigm, we assumed (although it was not a requirement) that participants would learn about the configuration of parts shared by all objects, as opposed to only individuating objects based on idiosyncratic features. Participants in the visual training paradigm certainly learned to use the configuration of parts to facilitate their recognition (Gauthier \&

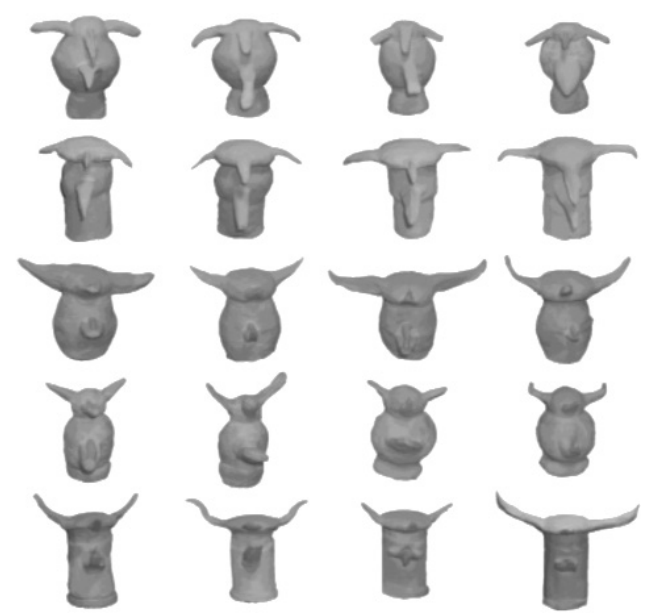

Figure 2. Clay 3-D Greebles-pictures of clay models of a subset of the Greebles. In each row are selected individuals from the same group. The top two rows are Ploks, and the bottom three rows are Glips. 


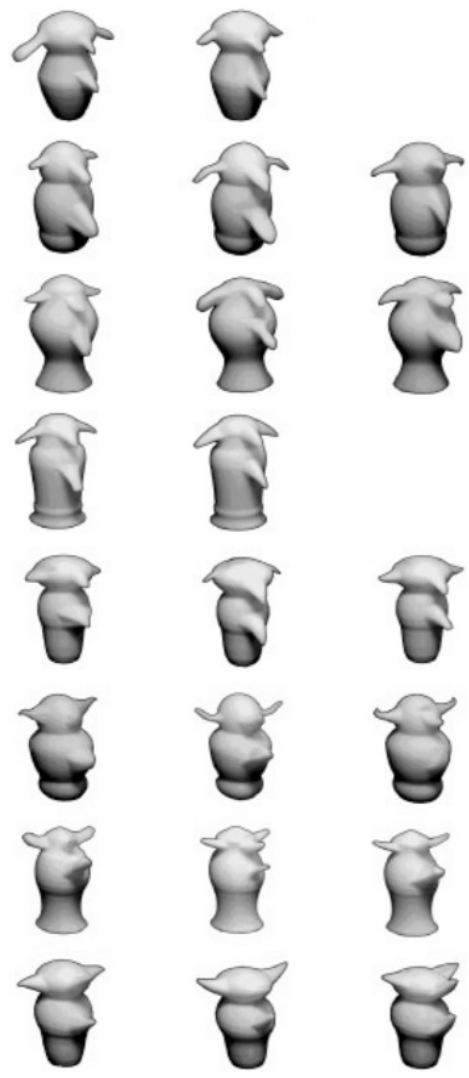

Figure 3. Plastic 3-D Greebles - pictures of plastic models of a subset of the Greebles created using rapid prototyping. In each row are selected individuals from the same group. The top five rows are Ploks, and the bottom three rows are Glips.

Tarr, 2002). It is possible that with the clay Greebles, idiosyncratic tactile features may have been introduced during sculpting, and that these features could be used to further individuate the Greebles, thus lessening the need to learn configurations. Consequently, imperfect reproduction of the Greebles may have caused changes in the learning strategy of the participants. A pilot study carried out with two participants suggested that haptic experience did not increase reliance on the configuration of parts. It was not possible from this pilot study to determine whether the lack of holistic processing was due to imperfections in the stimuli or provided evidence that haptic experience does not recruit holistic processing. Using a set of stimuli that did not have imperfections, however, could answer this question.

Plastic Greebles. Because accurate reproduction of the Greebles was deemed important for studying the effects of haptic expertise training, a second set of Greebles were built using a technology called rapid prototyping (Figure 3). Rapid prototyping is an emerging technology within the industrial design and engineering communities (for a brief review, see Amato, 2003). Earlier methods for realizing 3-D object models as solid, physical objects were both slow and costly, typically involving hand-coding to translate computer-aided design (CAD) models into instructions for a computer numerical control (CNC) milling machine. Advances in both solid modeling software and material science have enabled a different approach - rapid prototyping. The key idea behind this approach is to move the CAD-based 3-D model of an object directly to the rapid prototyping system, which then, in a relatively short time, builds a physical model of the object. Variations include the particular substrate used for the prototype (ranging from plaster of paris to aluminum) and whether individual parts are colored or not. At present, the technology is limited to relatively small objects: The largest 3-D volume that is possible on any rapid prototyping machine is approximately a $2 \times 2 \times 2 \mathrm{ft}$ cube (which, if completely filled, would consume an enormous amount of substrate); a more typical limit would be in the neighborhood of a cubic foot.

The actual process by which a rapid prototype of a Greeble is created can be summed up as " 3 -D printing." A 3-D CAD model of the object is carved up into a series of top-to-bottom slices, each slice approximating the cross-section of the object over a very small vertical distance (measured in 1,000ths of an inch). Each of these slices is effectively one "page" of a print run that describes the entire object: Imagine taking a series of such pages and stacking them so as to create a physical model. This is exactly what happens in rapid prototyping. However, the "ink" is a substance that either starts as a viscous liquid or can be temporarily converted to one (a plastic polymer in the case of the system we used). The rapid prototyping system we used (an FDM3000 system from Stratasys, Inc., Eden Prairie, MN) builds the object by printing the bottom slice with this substrate and then printing each successive slice, one on top of the other. As the substrate hardens, each slice bonds to adjacent slices, creating a solid, physical realization of the object in question - in our case, a Greeble. The actual prototypes are quite robust and have the feel of a machined, plastic part. Greebles were built with a mean height of $9 \mathrm{~cm}$ and a slice thickness of $0.254 \mathrm{~mm}$. Mean build time for each Greeble was approximately 10 hours.

Several challenges were involved in realizing the Greebles as physical objects. Most of these problems were specifically related to the original 3-D format in which the Greebles were created. Sadly, even more so than for 2-D images, there is no standard format for representing 3-D objects. Thus, there is no guarantee that one 3-D modeling program will be able to parse an object created using a different program. Moreover, translation from one 3-D format to another often produces geometry errors and rarely, if ever, preserves accurate surface properties (e.g., textures and colors). The Greebles were originally created in 1994-1995 with an early 3-D modeling application for Apple Macintosh personal computersAliasSketch! (a now-defunct product of Alias, Toronto). With the demise of AliasSketch!, the functionality of the Greeble set became increasingly diminished, and we eventually undertook the conversion of the Greebles 
from AliasSketch!'s esoteric native 3-D format (which relied almost exclusively on B-splines) to what has become the closest thing to a 3-D standard in the modeling world today, the 3DS format used by $3 \mathrm{ds}$ Max (Discreet, Montreal). Because of a wide variety of geometry errors that occurred during translation between these formats, conversion of the 80-object set required the better part of a year. Rendered images of the Greebles, plus 3-D models in the 3ds Max (formerly 3D Studio) format, are available at www.cog.brown.edu/ tarr/stim/Greebles-2-0symmetric.sit.

Nevertheless, a (near) standard format offers many advantages, not the least of which is portability to other 3-D modeling packages. Rapid prototyping systems are typically built around the STL file format, which was originally developed to support stereolithography (an early rapid prototyping technology). The two systems at the Department of Engineering at Brown University both rely on this format (one from Z Corporation, Burlington, MA, and the other from Stratasys). Having converted the Greeble set into 3ds Max's native (standard) format, it seemed that it would be fairly straightforward to create real Greebles by using 3ds Max to translate the models into the STL format, and 2-D rendered bitmaps of the Greebles were in fact easily created after conversion into the $3 \mathrm{ds}$ Max format. Rapid prototyping, however, places stricter demands on the veridical CAD model than does $2-\mathrm{D}$ rendering. For example, two adjacent planes may define a surface that can reflect a virtual light-source and therefore appears solid when rendered to a 2-D bitmap image. Those same two planes, however, may not be realizable as a physical entity if the planes are not actually numerically adjacent to each other. In other words, if there is no restriction that two surfaces must always intersect at edges and form solid models, models can be rendered but not built as prototypes. The newest versions of 3ds Max promote "good model topology" automatically for newly created models but do not enforce it for models that are converted from other formats. Therefore, a great deal of trial and error was required for hand tuning each converted Greeble model so that it would actually "print" using the rapid prototyping system. Topographical fit would not have been a problem if the Greebles had been designed originally in a solid modeling package that automatically verified geometry, such as $3 \mathrm{ds}$ Max.

\section{Apparatus}

The participants were seated at a table across from the experimenter and in front of a black fabric screen that blocked their view of the experimental area. The apparatus was fashioned so that participants were able to reach their arms under the occluding screen without it restricting their movement. There was a "rest position" for each hand, and the right-hand rest position was demarcated by a transparent plastic dome. The dome was positioned over a photodetector, into which shone an in- frared (IR) beam created using an IR laser diode module (Calpac Lasers, Model CP-TIM-201-5D-650; www. calpaclasers.com) that was suspended above the detector. Moving the right hand from the rest position revealed the photodetector, which in turn sent a signal through a custom-built circuit. Output from the circuit was split: One signal went to a computer that recorded accurate response times, and the other went to the video recording equipment.

Video was recorded directly to digital video files (QuickTime movies) on an external hard drive by means of a FireStore direct-to-edit recording device (Focus Enhancements, Campbell, CA; www.focusinfo.com). The FireStore device also received an input from the IR beam circuit by means of its own general purpose interface (GPI; Model RS232C) port. The GPI port was set to pause and unpause the record mode. At the beginning of each experiment, the FireStore was put into record mode and paused. When the participant's hand broke the IR beam while making a movement toward the Greeble, the FireStore was unpaused and began recording a video clip. When the participant's hand broke the IR beam while returning to the rest position, the FireStore was paused once again. Therefore, only the exploratory hand movements were captured, making postprocessing of the video much less time consuming. To archive the video files, they were first converted to a compressed MPEG-4 format using QuickTime Pro (www.apple.com/quicktime) on a Macintosh computer, which compressed their size by approximately six times.

\section{REFERENCES}

Amato, I. (2003). Instant manufacturing. Technology Review, 106, 56-62. Amedi, A., Jacobson, G., Hendler, T., Malach, R., \& Zohary, E. (2002). Convergence of visual and tactile shape processing in the human lateral occipital complex. Cerebral Cortex, 12, 1202-1212. ERnst, M. O., \& BANKS, M. S. (2002). Humans integrate visual and haptic information in a statistically optimal fashion. Nature, 415, 429-433.

Ernst, M. O., Banks, M. S., \& Bülthoff, H. H. (2000). Touch can change visual slant perception. Nature Neuroscience, 3, 69-73.

Friedman, A., Spetch, M. L., \& LANK, I. (2003). An automated apparatus for presenting depth-rotated three-dimensional objects in human and animal object recognition research. Behavior Research Methods, Instruments, \& Computers, 35, 343-349.

Gauthier, I., \& TARr, M. J. (1997). Becoming a "Greeble" expert: Exploring mechanisms for face recognition. Vision Research, 37, 16731682.

GAUTHIER, I., \& TARR, M. J. (2002). Unraveling mechanisms for expert object recognition: Bridging brain activity and behavior. Journal of Experimental Psychology: Human Perception \& Performance, 28, 431-446.

Gauthier, I., TARr, M. J., Anderson, A. W., Skudlarski, P., \& Gore, J. C. (1999). Activation of the middle fusiform "face area" increases with expertise in recognizing novel objects. Nature Neuroscience, $\mathbf{2}$, 568-573.

Gauthier, I., Tarr, M. J., Moylan, J., Skudlarski, P., Gore, J. C., \& Anderson, A. W. (2000). The fusiform "face area" is part of a network that processes faces at the individual level. Journal of Cognitive Neuroscience, 12, 495-504.

Gauthier, I., Williams, P., TARr, M. J., \& Tanaka, J. (1998). Training "greeble" experts: A framework for studying expert object recognition processes. Vision Research, 38, 2401-2428. 
Hadjikhani, N., \& Roland, P. E. (1998). Cross-modal transfer of information between the tactile and the visual representations in the human brain: A positron emission tomographic study. Journal of Neuroscience, 18, 1072-1084.

Heller, M. A. (2002). Tactile picture perception in sighted and blind people. Behavioural Brain Research, 135, 65-68.

James, T. W., Humphrey, G. K., Gati, J. S., Servos, P., Menon, R. S., \& Goodale, M. A. (2002). Haptic study of three-dimensional objects activates extrastriate visual areas. Neuropsychologia, 40, 17061714.

Kilgour, A. R., \& Lederman, S. J. (2002). Face recognition by hand. Perception \& Psychophysics, 64, 339-352.

Klatzky, R. L., \& Lederman, S. J. (1995). Identifying objects from a haptic glance. Perception \& Psychophysics, 57, 1111-1123.

Klatzky, R. L., Lederman, S. J., \& Metzger, V. A. (1985). Identifying objects by touch: An "expert system." Perception \& Psychophysics, 37, 299-302.

LEDERMAN, S. J., \& KLATZKY, R. L. (1993). Extracting object properties through haptic exploration. Acta Psychologica, 84, 29-40.

Prather, S. C., \& Sathian, K. (2002). Mental rotation of tactile stimuli. Cognitive Brain Research, 14, 91-98.

Rossion, B., Gauthier, I., Goffaux, V., Tarr, M. J., \& Crommelinck, M. (2002). Expertise training with novel objects leads to left-lateralized facelike electrophysiological responses. Psychological Science, 13, 250-257.

Servos, P., Lederman, S., Wilson, D., \& Gati, J. (2001). fMRI-derived cortical maps for haptic shape, texture, and hardness. Cognitive Brain Research, 12, 307-313.
Zangaladze, A., Epstein, C. M., Grafton, S. T., \& Sathian, K. (1999). Involvement of visual cortex in tactile discrimination of orientation. Nature, 401, 587-590.

\section{ARCHIVED MATERIALS}

The following materials and links may be accessed through the Psychonomic Society's Norms, Stimuli, and Data archive, http://www. psychonomic.org/archive/.

To access these files or links, search the archive for this article using the journal (Behavior Research Methods), the first author's name (James), and the publication year (2005).

FILE: James-BRM-2005.zip.

DESCRIPTION: The compressed archive file contains two folders:

The Images folder contains the 2-D rendered bitmap images of the Greeble models in TIFF format.

The Models_3DS folder contains the 3-D Greeble models in the more portable 3D Studio (3DS) format.

The file names follow the conventions "GF FI.3ds" for models and "GF FI-vV.tif" for images. The $G$ represents the "gender," either (m)ale or (f)emale, defined by orientation of parts; $F$ represents the family, numbered 1 through 5 , defined by body shape; $I$ represents the individual, numbered 1 through 10, defined by the shape of the parts. For images, $V$ represents the view, numbered 1 or 2; these two views are transformations formed by rotating about the vertical axis.

AUTHOR's E-MAIL ADDRESS: michael_tarr@brown.edu.

AUTHOR's WeB SITE: http://www.cog.brown.edu/ tarr. 